Special Issue of the 7th International Advances in Applied Physics and Materials Science (APMAS 2017)

\title{
Thermal, Electrical, and Mechanical Properties of Various Thermal Conductive Powder Filled Polyamide 6 Composite Materials for Thermal Management Applications
}

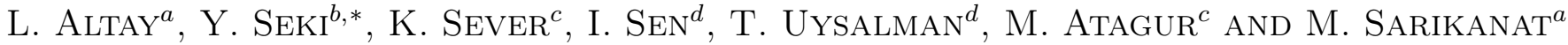 \\ ${ }^{a}$ Ege University, Mechanical Engineering Department, Bornova, Izmir, Turkey \\ ${ }^{b}$ Dokuz Eylul University, Faculty of Science, Buca, Izmir, Turkey \\ ${ }^{c}$ Izmir Katip Celebi University, Faculty of Engineering and Architecture, Çigli, Izmir, Turkey \\ ${ }^{d}$ Izmir Egitim Saglik Sanayi Yatirim A.S., Turgutlu, Manisa, Turkey
}

\begin{abstract}
The aim of this study is to develop composites with high thermal conductivity by employing the thermophysical properties of aluminum nitride (AlN), hexagonal boron nitride (hBN), and synthetic graphite (SG). AlN, hBN, and SG filled polyamide 6 (PA) composites were prepared using a co-rotating twin-screw extruder and injection molding. Changes in thermal, mechanical, and electrical properties of the composites were investigated. The highest in-plane and through-plane thermal conductivity values were obtained with $50 \mathrm{wt} . \% \mathrm{SG}$ and $50 \mathrm{wt} . \%$ AlN loadings, respectively. Mechanical strength of PA decreased for all filler loadings. Electrical conductivity results showed that AlN and hBN filled PA composites behave as electrical insulators. On the contrary, SG loading led to electrically conductive PA composites.
\end{abstract}

DOI: 10.12693/APhysPolA.134.200

PACS/topics: polyamide composites, thermal management, electrical properties, mechanical properties

\section{Introduction}

Thermoplastics show electrical insulating behavior, which is favorable for such applications as insulating parts in electrical engineering and electronics. In terms of these applications, the main disadvantage of using thermoplastics is their low thermal conductivity. The thermal conductivity is well below $0.4 \mathrm{~W} / \mathrm{mK}$ for many thermoplastics and this leads to the accumulation of heat in the insulation parts [1].

For improving the thermal conductivity of a polymeric material, polymer matrices are usually filled with thermally conductive fillers like metal powders (copper, steel, iron, aluminum, etc.), ceramic powders (boron nitride, aluminum nitride, aluminum oxide) and carbonbased materials (carbon fiber, synthetic graphite, carbon black). By using AlN $[2,3]$ and $\mathrm{hBN}[3,4]$ as thermally conducting but electrically insulating fillers, thermoplastic based composites can become thermally conducting while remaining electrically insulating behaviour. However, carbon (graphene, carbon nanotubes, graphite, synthetic graphite) [5-8] and metal [9-11] fillers are usually used for thermally and electrically conductive thermoplastic composites.

Thermally conductive polymeric composites find applications in many industries such as electronics, power generation, thermal coatings, cooling devices, heat conduction of functional elements, computer chassis, lithium ion batteries, aviation, and many other industries $[12,13]$.

*corresponding author; e-mail: yoldas.seki@deu.edu.tr
The main goal of this study is to determine the effects of different thermal conductive fillers on the morphological, mechanical, electrical, and thermal properties of thermally conductive filled PA composites. For this study, three different thermally conductive fillers of SG, $\mathrm{hBN}$, and AlN were used. PA based formulations containing $50 \mathrm{wt} . \%$ of conductive fillers were extruded and injection molded into test specimens.

\section{Materials and methods}

PA with the trade name of Volgamid 2.7 was supplied by İzmir Eğitim Sağlık Sanayi Yatırım AS, Turkey. The density of PA is $1.15 \mathrm{~g} / \mathrm{cm}^{3}$. The fillers used in this study are Thermocarb TC series SG with a mean particle size of $50 \mu \mathrm{m}$ (Asbury, NJ), hBN with a mean particle size of $50 \mu \mathrm{m}$ (H.C. Starck) and AlN with a mean particle size of 7-11 $\mu \mathrm{m}$ (H.C. Starck). The thermal conductivity of SG is about $600 \mathrm{~W} / \mathrm{mK}$ in-plane and $6 \mathrm{~W} / \mathrm{mK}$ through-plane [14]. The thermal conductivity of $\mathrm{hBN}$ is about $600 \mathrm{~W} / \mathrm{mK}$ in plane and between $2-30 \mathrm{~W} / \mathrm{mK}$ in through-plane direction [15]. The thermal conductivity of AlN is about $170-380 \mathrm{~W} / \mathrm{mK}$ in-plane and throughplane directions [16].

\subsection{Composite preparation}

50 wt.\% AlN, hBN, and SG filled PA composites (PA50SG, PA-50AlN and PA-50hBN) were prepared using a twin screw extruder (Leistritz Extruder Corporation, Model ZSE 27) with $300 \mathrm{rpm}$ at barrel temperatures of $220-250{ }^{\circ} \mathrm{C}$. Then, composites were injection molded into test specimens with injection molding machine (Bole model BL90EK). 


\subsection{Characterization methods}

Thermal stability of PA and PA-50SG, PA-50AlN, PA-50hBN composites was investigated by thermogravimetric analysis using a TGA analyzer (TA Instruments Inc., TGAQ500) at a heating rate of $10^{\circ} \mathrm{C} / \mathrm{min}$ in the range of $30-600^{\circ} \mathrm{C}$. DSC analyzer (TA Instruments Inc., DSCQ20) was used for differential scanning calorimetry analysis. Measurements were carried out in the temperature range between 50 and $200^{\circ} \mathrm{C}$ at a rate of $10^{\circ} \mathrm{C} / \mathrm{min}$.

In-plane and through-plane thermal conductivity measurements of PA, PA-50SG, PA-50AlN, and PA-50hBN were performed by using Flash Diffusivity Analyzers (Discovery Xenon Flash DXF 200) according to ASTM 1461. Surface electrical resistivity of PA and PA based composites was measured using ASTM-D-257-9 standard with 6517B/E Keithley Electrometer/High Resistance Meter.

The tensile properties of PA and PA composites with SG, AlN and hBN were investigated using a Shimadzu Autograph AG-IS Series universal testing machine equipped with a video extensometer system (Shi- madzu Non-contact Video Extensometer DVE-101/201) at a crosshead speed of $50 \mathrm{~mm} / \mathrm{min}$ according to ASTM standard D638-10.

Three-point bending tests were conducted to characterize the flexural properties of the PA and PA composite plates by following the ASTM D790 standard. The tests were carried out at a constant crosshead speed of $2 \mathrm{~mm} / \mathrm{min}$ and span length of $32 \mathrm{~mm}$. The fracture surfaces of PA, PA-50SG, PA-50AlN, and PA$50 \mathrm{hBN}$ specimens were examined using a scanning electron microscope (SEM) (Carl Zeiss 300VP, Germany) operated at $5 \mathrm{kV}$.

\section{Results and discussion}

The thermal degradation behavior of PA and its composites is tabulated in Table I. Maximum degradation temperature, $T_{\max }$, values are obtained from the first derivative of TGA curves. $T_{\max }$ of PA was obtained to be $443{ }^{\circ} \mathrm{C}$. The introduction of varying types of fillers alters the thermal decomposition mechanism of PA matrix.

Thermal properties of PA, PA-50SG, PA-50AIN and PA-50hBN composites

TABLE I

\begin{tabular}{l|c|c|c|c|c|c}
\hline \hline & $T_{\max }\left[{ }^{\circ} \mathrm{C}\right]$ & $\begin{array}{c}\text { Degraded } \\
\text { weight }[\%]\end{array}$ & $T_{\mathrm{m}}\left[{ }^{\circ} \mathrm{C}\right]$ & $T_{\mathrm{c}}\left[{ }^{\circ} \mathrm{C}\right]$ & $\Delta H_{\mathrm{m}}[\mathrm{J} / \mathrm{g}]$ & $X_{\mathrm{c}}[\%]$ \\
\hline PA & 443 & 99.9 & 222 & 179 & 57.7 & 25.1 \\
PA-50SG & 453 & 49.6 & 221 & 195 & 33.8 & 29.4 \\
PA-50AlN & 453 & 49.0 & 221 & 188 & 31.6 & 27.5 \\
PA-50hBN & 455 & 49.5 & 220 & 192 & 60.2 & 52.3
\end{tabular}

When 50 wt.\% SG, AlN, or hBN were added into $\mathrm{PA}, T_{\max }$ slightly increased to 453,453 , and $455^{\circ} \mathrm{C}$, respectively compared to that of $\mathrm{PA}$. From Table I, PA-50hBN composite has the highest $T_{\max }$ among all studied composites. This can be ascribed to fact that highly thermally conductive fillers can dissipate heat during heating and thus delay the degradation. This led to a shift of $T_{\max }$ to a higher value, which indicates an increase in thermal stability of PA composites filled with SG, AlN or hBN.

TGA results also show that mass losses are 49.6, 49.0 and $49.5 \%$ for PA-50SG, PA-50AlN, and PA-50hBN, respectively. The mass loss value of composites agrees well with mass loss values predicted based on filler weight fraction, which suggests that filler is dispersed uniformly in PA matrix.

An increasing trend in the crystallization temperatures, $T_{\mathrm{c}}$, of different composites of PA can be seen in Table I. Fillers do not have a noticeable effect on the melting temperature, $T_{\mathrm{m}}$, of composites. The DSC investigations of $\mathrm{PA}$ and its composites indicate that $\Delta H_{\mathrm{m}}$ value, which is related to the degree of crystalinity $X_{\mathrm{c}}$, is the highest for PA- $50 \mathrm{hBN}$. The value of $X_{\mathrm{c}}$ of PA in the composites increases by $29.4,27.5$, and $52.3 \%$ with 50 wt.\% loadings of $\mathrm{SG}, \mathrm{AlN}$, and $\mathrm{hBN}$, respectively. The melting enthalpy of a $100 \%$ pure crystalline PA is $230 \mathrm{~J} / \mathrm{g}$, a commonly accepted average value for PA [17].
Thermal conductivity values of polymer composites mainly depend on the conductivity of polymer matrix and fillers, interfacial interactions between polymer matrix and fillers, and the anisotropic thermal properties of the filler materials. Measured in-plane and through-plane thermal conductivity results for PA and its composites are given in Fig. 1a and b. As is seen from both figures, an obvious increase in the thermal conductivity of composites was achieved by introducing SG, AlN, and hBN into PA. The in-plane thermal conductivity values increased greatly compared to through-plane conductivities.

It is known that thermal conductivities of SG and hBN along in-plane direction are much higher than those in the through-plane direction due to intrinsic anisotropy of these platelet-structured materials. The measured in-plane thermal conductivity values are 7.6, 2.7, and $3.6 \mathrm{~W} / \mathrm{mK}$ for PA-50SG, PA-50AlN, and PA-50hBN, respectively. From Fig. 1a, PA-50SG exhibits higher inplane thermal conductivity than those of PA-50hBN and PA-50AlN. The SG loading enhances the in-plane thermal conductivity of PA by approximately 24 times. It can be seen that in-plane thermal conductivity of PA$50 \mathrm{hBN}$ is very high compared to through-plane conductivity, which indicates that the hBN platelets are highly in-plane oriented in the PA matrix. This can be explained by the lubricious properties of hBN platelets which make them easily oriented along the flow during the injection 

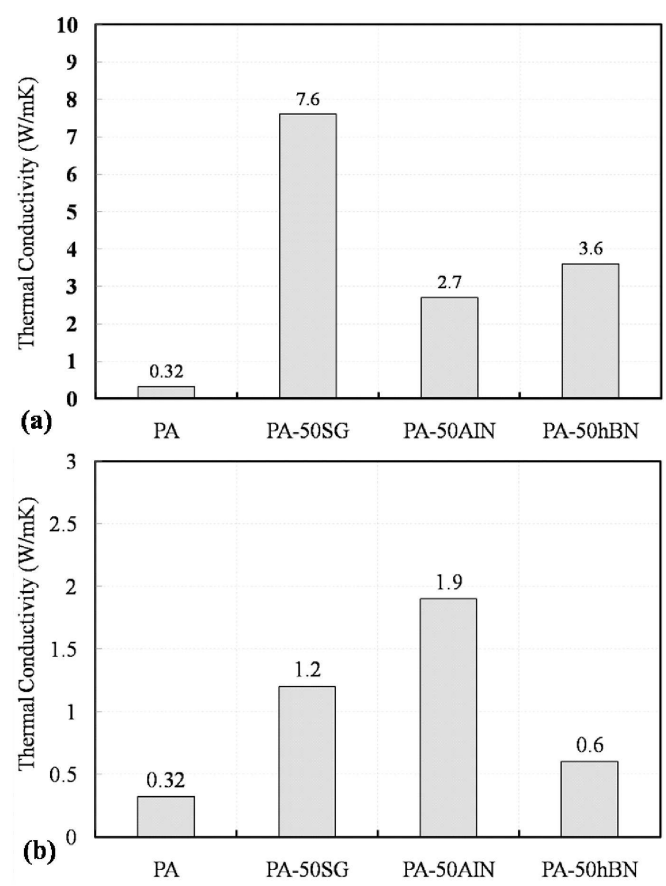

Fig. 1. In-plane (a) and through-plane (b) thermal conductivity of PA, PA-50SG, PA-50AlN, and PA$50 \mathrm{hBN}$.

process. Besides, it is known that the nitrogen and boron atoms are held together by strong covalent bonds within each hBN layer, between which weak Van der Waals force occurs [18]. Considerable anisotropies of the shape and of the thermal conductivity of $\mathrm{hBN}$ and the $\mathrm{hBN}$ filled PA are strongly dependent on the orientation of the hBN [19].

Figure 1b shows through-plane thermal conductivities of PA composites filled with SG, AlN and hBN. The thermal conductivity of the PA is around $0.32 \mathrm{~W} / \mathrm{mK}$. The through-plane thermal conductivity of PA increases with the introduction of fillers into PA, as seen in Fig. 1b. Incorporation of SG, AlN and hBN into PA led to about $275 \%, 493 \%$, and $87 \%$ increases in through-plane thermal conductivities, respectively. The highest through-plane conductivity was achieved when AlN was added to the PA matrix. This result can be ascribed to wurtzite structure of AlN, which is beneficial to form thermal pathways in polymer composite along the through-plane direction.

Figure $2 \mathrm{a}$ and $\mathrm{b}$ show the effects of addition of $\mathrm{SG}$, $\mathrm{AlN}$, and hBN into PA matrix on the tensile and flexural strengths of PA composites, respectively. The tensile and flexural strengths of PA decreased with the addition of SG, AlN, and hBN. When comparing the tensile and flexural strengths of PA composites with different types of filler, SG and hBN performed quite similarly in both cases.

As it is seen from Fig. 2a, the amount of decrease in tensile strength is about the same when $\mathrm{SG}$ or $\mathrm{hBN}$ are used as fillers. However, composite containing 50 wt.\%
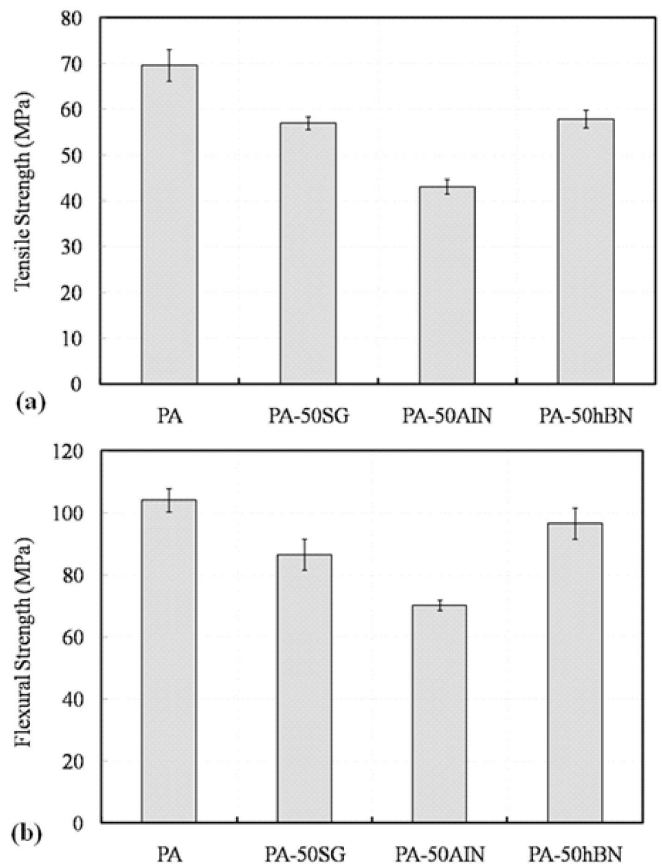

Fig. 2. Tensile strength (a) and flexural strength (b) values of PA, PA-50SG, PA-50AlN, and PA-50hBN.

AlN shows the highest decrease in tensile strength compared to PA-50SG and PA-50hBN composites. This can be ascribed to poor dispersion of AIN particles. In other words, weak adhesion between polymer and AlN within the composite material may reduce the overall strength of the material.

Similar phenomena were observed in the flexural strength results. From Fig. 2b, a reduction of flexural strength was observed at 50 wt.\% AlN loading, whereas flexural strengths of PA-50SG and PA-50hBN composites were similar to that of pristine PA. One possible explanation of the reduced strength is that the presence of weak adhesion between AlN and PA yields poor transfer of stress between the polymer matrix and the fillers.

Surface resistivity values of PA, PA-50AIN, and PA$50 \mathrm{hBN}$ were measured to be $4.96 \times 10^{13}, 1.49 \times 10^{13}$, and $6.39 \times 10^{13} \mathrm{Ohm} / \mathrm{sq}$., respectively, which indicates electrically insulating polymeric composites. However surface resistivity of PA-50SG, which was lower than $10^{3} \mathrm{Ohm} / \mathrm{sq}$., shows that this is an electrically conductive material.

SEM images of PA-50SG, PA-50AlN, and PA-50hBN are shown in Fig. 3a, b, and c, respectively. In SEM images of PA-50SG (Fig. 3a) and PA-50hBN (Fig. 3c) the platelet morphologies of $\mathrm{SG}$ and $\mathrm{hBN}$ are noticeable. Heat transfer takes place through the linkages between the platelets [20]. In Fig. 3b, bare AlN particles of micron size and holes are seen. Particle pull-out can be seen, which is due to poor interface between AIN particles, present in different sizes, and the PA matrix. 

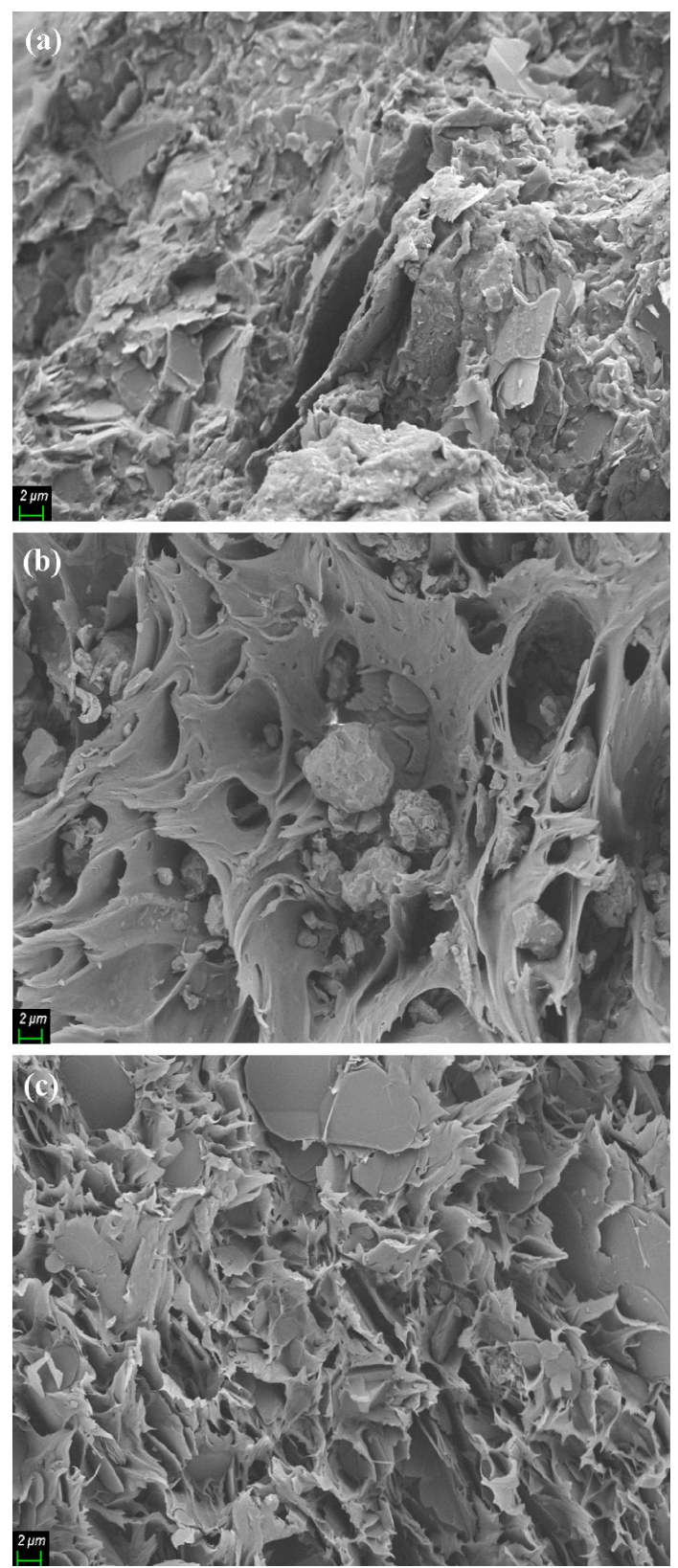

Fig. 3. SEM images of PA-50SG (a), PA-50AlN (b) and PA-50hBN (c).

\section{Conclusions}

The in-plane thermal conductivity values of PA6, PA-50SG, PA-50AlN, and PA-50hBN were obtained to be $0.32,7.6,2.7$, and $3.6 \mathrm{~W} / \mathrm{mK}$, respectively. These values indicate about 24, 8, 11 times higher in-plane thermal conductivity for PA-50SG, PA-50AlN, and PA-50hBN, respectively, compared to PA. Higher heat transfer for PA-50SG and PA-50hBN can be explained by the linkages between platelets, which were observed in SEM micrographs. Incorporation of SG, AlN and hBN into the PA increases through-plane conductivities by $275 \%, 493 \%$, and $87 \%$, respectively. While PA-50AIN and PA-50hBN are electrically insulating, the PA-50SG shows electrical conductivity. PA containing AlN of $50 \%$ by weight shows the highest decrease in tensile strength and flexural strength, compared to PA-50SG and PA$50 \mathrm{hBN}$ composites. Incorporation of SG, AlN, and hBN into PA does not affect the melting temperature of PA, however, it increases the crystallization temperature and crystallization degree of PA.

\section{References}

[1] B. Li, R. Li, Y. Xie, J. Mater. Sci. 52, 2524 (2017).

[2] L. Li, D.D.L. Chung, J. Electron. Mater. 23, 557 (1994).

[3] Y. Xu, D.D.L. Chung, Compos. Interf. 7, 243 (2000).

[4] N. Tsutsumi, N. Takeuchi, T. Kiyotsukuri, J. Polym. Sci. B Polym. Phys. 29, 1085 (1991).

[5] D.L. Gaxiola, M.M. Jubinski, J.M. Keith, J.A. King, I. Miskioglu, J. Appl. Polym. Sci. 118, 1620 (2010).

[6] E.H. Weber, M.L. Clingerman, J.A. King, J. Appl. Polym. Sci. 88, 112 (2003).

[7] R.A. Hauser, J.A. King, R.M. Pagel, J.M. Keith, J. Appl. Polym. Sci. 109, 2145 (2008).

[8] Y. Agari, T. Uno, J. Appl. Polym. Sci. 30, 2225 (1985).

[9] A.S. Luyt, J.A. Molefi, H. Krump, Polym. Degrad. Stabil. 91, 1629 (2006).

[10] A. Boudenne, L. Ibos, M. Fois, J.C. Majeste, E. Gehin, Compos. A - Appl. Sci. Manuf. 36, 1545 (2005).

[11] S.N. Maiti, K. Ghosh, J. Appl. Polym. Sci. 52, 1091 (1994).

[12] T. Tanaka, M. Kozako, K. Okamoto, J. Int. Council Electr. Engin. 2, 90 (2012).

[13] X.Y. Huang, P.K. Jiang, T. Tanaka, IEEE Electrical Insulation Magazine 27, 8 (2011).

[14] E. Pop, V. Varshney, A.K. Roy, MRS Bulletin 37, 1273 (2012).

[15] M. Kawaguchi, S. Kuroda, Y. Muramatsu, J. Phys. Chem. Sol. 69, 1171 (2008).

[16] A. Franco, D.J. Shanafield, J. Mater. Sci. Mater. Electron. 16, 139 (2005).

[17] Y.P. Khanna, W.P. Kuhn, J. Polym. Sci. B: Polym. Phys. 352219 (1997).

[18] M. TabkhPaz, S. Shajari, M. Mahmoodi, D.-Y. Park, H. Suresh, S.S. Park, Compos. B: Engin. 100, 19 (2016).

[19] Z. Lin, A. McNamara, Y. Liu, K.-s. Moon, C.P. Wong, Compos. Sci. Technol. 90, 123 (2014).

[20] G. Wypych, Handbook of Fillers, ChemTec Publishing, Toronto 2016. 